\title{
The Interoperability between Different e-Business Specifications ${ }^{1}$
}

\author{
Peng Liang, Keqing He, Bing Li, Jin Liu \\ State Key Lab of Software Engineering, Wuhan University, \\ Wuhan 430072, Hubei, P.R.China \\ liangp@sklse.org
}

\begin{abstract}
The interoperability among the e-Business solutions is the key issue for the implementation of seamless e-Business, and the interoperability of the eBusiness solutions are determined by the e-Business specification on which the e-Business solution are implemented. In this paper, the analysis is performed for the technical architecture comparison between cnXML and ebXML, the de facto e-Business standards in China and worldwide. The interoperability assurance layers between different e-Business specifications are proposed, namely the data layer, the semantic layer and process layer. We focus on the interoperability of data layer, namely the messaging service layer in this research, and through the XML schema comparison between cnMS and ebMS, we propose the interoperability test levels and assurance method for the e-Business messaging service layer.
\end{abstract}

Keywords: e-Business specification; cnXML; ebXML; interoperability

\section{Introduction}

Interoperability is a quite familiar and ambiguous concept in computer science. Normally we look on it in two aspects - hardware and software. The interoperability problem for software is more complex and difficult to assure than hardware. With the popularization of Internet and distributed computing, all kinds of information with different format and structure in computer system are required to be integrated seamlessly and transparently. This requirement raises the issue of interoperability. The Interoperability Assurance (IA) of e-Business solutions is the application of interoperability in practical domain.
e-Business is always a hot topic in business field since its appearance with EDI system in 1960s, while the high implementation cost is the biggest obstacle for SME (Small and Medium Enterprises) to come in and play [1]. With the introduction of XML (eXtensible Markup Language), the infrastructure mechanism of eBusiness technology has been totally changed. The business data and process can be both specified formally in XML, and more and more XML-based eBusiness specifications appeared in the last few years, such as ebXML, BizTalk, RosettaNet, cXML [1], and cnXML [2], the e-Business specification for the Chinese market. These different e-Business specifications will co-exist in a long time due to the industrial and regional protectionism, while the global e-Business environment requires the mutual understanding between e-Business solutions which are implemented based on different e-Business specification. Consequently, the IA between different e-Business specifications is the key issue for spreading e-Business technology around the world. Currently, the research on the interoperability between the different eBusiness solutions based on the same e-Business specification has been carried out [3], while less effort is put on the research on the interoperability between different e-Business specifications. In this paper, we select two e-Business specifications cnXML and ebXML, which are defined by China and ISO respectively for the interoperability research.

This paper is composed of the following sections. Section 2 describes the general concept of interoperability of software solutions in details, with special focus on the interoperability of e-Business solutions. Section 3 describes the common technical architecture of e-Business specifications, and analyzes the interoperability of e-Business specifications in details. Section 4 presents the interoperability

\footnotetext{
${ }^{1}$ This research project was supported by the National Natural Science Foundation of China under Grant No. 60373086; Wuhan Science \& Technique Key Project under Grant No. 20021002043; Open Foundation of SKLSE under Grant No. 03-03; the Provincial Natural Science Foundation of Hubei under Grant No. 2002ABB037.
} 
assurance method for the messaging service. Section 5 concludes this research with future work.

\section{The Software Interoperability}

In the real enterprise computing environment, we have to face the complex system which is composed of different operating systems, databases, and applications, etc. They communicate with each other in different programming language, data structure and interface which are the common characteristics of the heterogeneous system [4]. The IA of these heterogeneous systems is the most fundamental problem we have to solve other than security, functionality, QoS, etc. Interoperability has become the key issue for the development of information system infrastructure.

In [5], the general definition of Software Interoperability is: The ability of software system to share information among different computing systems, networks, operating systems and applications. The IA of software systems can only be achieved through standardized test and certification process.

To the filed of e-Business solutions, we define the interoperability as follows: The solution can be implemented by following certain specification (e.g. cnXML or ebXML) or standard, and the function of the implementation is independent from specific programming language and operating system. The implemented functionality for the interaction between solutions is checkable.

\section{Interoperability between different e- Business Specifications}

As discussed in session 1, the interoperability of the e-Business solutions is determined by the interoperability of the e-Business specifications. In this research, we select two e-Business specifications cnXML and ebXML, which are defined by China and ISO respectively for the interoperability research. With the trend of China economy merging into globalization process, we argue that this research will have much more practical meaning. In this section, the common technical architecture of e-Business specification is analyzed, and the interoperability assurance layers are specified according to the common technical architecture.

\subsection{Common Technical Architecture of e- Business Specification}

There are many kinds of e-Business specifications available presently, and most of them are XML-based, which means their description mechanism is the same. These specifications also have the similar common technical architecture, which is compatible with the technical architecture of Web Service [6]. These specifications are normally composed of a set of specifications, including Messaging Service specification, which defines the message format and delivery rules; Registry \& Repository specification, which defines the registry information model and repository model; Business Process specification, which defines the frequently used business process; Business Terms specification, which defines the common used business terms required in the business process; and Common Document Specification, Trading Partner Profile/Agreement specification, these complementary specifications [7]. The specification set of cnXML and ebXML are almost the same except that cnXML includes a Common Document specification, which defines the XML schema of the common used document types, such as invoice and order form.

cnXML and ebXML follow the modularized architecture, which means each module of the specification can be implemented individually without the support of other modules, such as messaging service, registry service, etc. This advantage can be beneficial to the SME to deploy their e-Business solutions step by step, and keep the extensibility of the system.

\subsection{Interoperability Assurance Layers between different e-Business Specifications}

Interoperability Assurance layers between different e-Business Specifications are similar with the seven layers in the network protocol. The Interoperability of each lower layer should be ensured before the upper layer. According to the Common Technical Architecture of e-Business Specification, the Interoperability Assurance layers between different eBusiness Specifications are divided into three layers: data, semantic, and process layers as shown in Figure 1. In this figure, data layer corresponds to messaging service specification; semantic layer corresponds to business term specification, and process layer corresponds to business process specification. The relationship of these three layers is that the IA of lower layer is the basic for the IA of upper layer. Messaging service specification defines the basic format and delivery mechanism of business message, such as sending order message. This layer is the lowest layer in the technical architecture, consequently it is the fundamental layer for the IA of the whole specification; Business term specification defines the common used business terms, such as Order, Invoice, 
etc, but the communication of content can only be achieved by messaging service; Business Process specification defines the frequently used business process which is composed of a set of message sequence in fixed order, such as Ordering-ShippingInvoicing process. The business process employs the business terms which are defined in business term specification, so this layer locates in the highest layer of the whole specification.

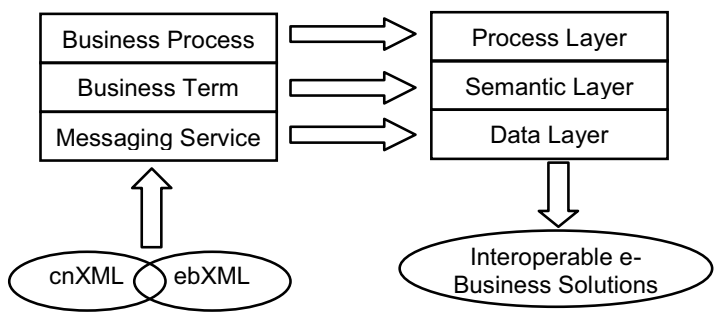

Figure 1. Interoperability assurance layer between different e-Business specifications

\section{Interoperability of Messaging Service Layer}

As shown in Figure 1, Messaging service layer is the lowest layer in the Interoperability Assurance layer between different e-Business Specifications. This layer ensures that the message can be delivered between business partners reliably and securely, which is the basic requirements for the e-Business, consequently it is the fundamental layer for the IA of the whole specification. This section will focus on the Interoperability of Messaging Service layer.

\subsection{Comparison between cnMS and ebMS}

Messaging Service specification defines the message format, and the message is described by XML, so the message format is actually the XML schema defined in the specification. The message format of cnMS and ebMS are both based on SOAP specification, including header container and payload container [8]. In Figure 2, the XML schema comparison between cnMS and ebMS is demonstrated with the elements mapping relationship $[9,10]$. The detailed analysis is given below.

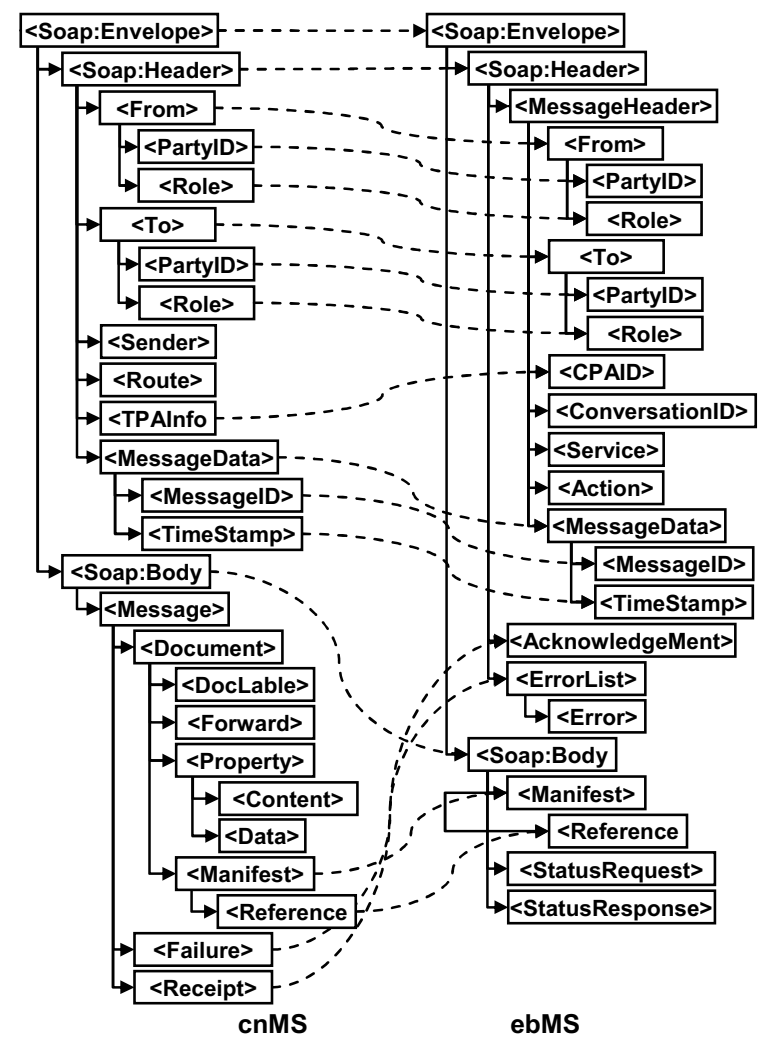

Figure 2. Comparison of XML schema between cnMS and ebMS

In the header container, the XML Schema of cnMS and ebMS both include $<$ Header $>$ element and $<$ Body $>$ element.

\subsubsection{The Comparison of $<$ Header $>$ element}

From the structure point of view, the $<$ Header $>$ element of cnMS includes the main description subelement of the message, and in ebMS, the $<$ Header $>$ element only includes a $<$ MessageHeader $>$ subelement to manage the main description element of the message.

From the function point of view, the $<$ Header $>$ element of cnMS provides the information for message certification, message route, and the attribute of the message data. In the $<$ Header $>$ element of ebMS, it describes the basic business attribute, such as message routing, security, and management information.

As for the sub-element definition of $<$ Header $>$ element of cnMS and ebMS, the $<$ From $>,<\mathrm{To}>$, $<$ MessageData $>$ are the same. The names of some other elements are not the same, but their semantic is the same, and can be mapped between cnMS and ebMS. For example, the <TPAInfo $>$ element in cnMS can be mapped to the $<$ CPAID $>$ element in ebMS. 
There are also some different elements: In cnMS, $<$ Sender $>$ element specify the current message sender, $<$ Route $>$ element specify the message route information. When a receiver receives a cnMS message and wants to send it to another receiver, it should add the $<$ Sender $>$ element information to the $<$ Route $>$ element in the new message, and marks its ID in the $<$ Sender $>$ element of the new message. In ebMS, the message uses the $<$ ConversationID $>$ element to specify the conversation session to which current message belongs to, and normally a conversation is composed of multiple interactive messages to complete certain business function. The $<$ Service $>$ element specifies the service type provided by receiving party. The $<$ Action $>$ element specifies the activity executed or processing type by this message.

\subsubsection{The Comparison of $<$ Body $>$ element}

From the function point of view, the $<$ Body $>$ element of cnMS is responsible to record the content of the message, namely payload. The content of the message can be classified into three types: document, failure processing, and receipt processing. The $<$ Body $>$ element of ebMS describes processing information for the payload, such as message status information.

Due to the function difference of $<$ Body $>$ element, the structure of the $<$ Body $>$ element is quite different between cnMS and ebMS. In cnMS, $<$ Body $>$ element only includes one <Message $>$ sub-element. This element manages the payload and the attribute of payload with $<$ Failure $>$ and $<$ Receipt $>$ sub-element to describe the failure and receipt processing. In ebMS, $<$ Body $>$ element is responsible directly the payload manifest and message status information. By the comparison, the biggest difference between cnMS and ebMS is that: cnMS includes the payload information in the <Envelope $>$ element; on the contrary, ebMS excludes the payload information from the $<$ Envelop $>$ element.

For the sub-element definition, in cnMS, $<$ Document $>$ element includes the concrete content delivered by the message, namely the payload. $<$ Failure $>$ element describes the error message, and is send to the sender by the failure message receiver. $<$ DocLabel $>$ element specifies the document name. $<$ Forward $>$ element specifies the forwarding address. $<$ Property $>$ element specifies the document type. $<$ Content $>$ element includes the XML format content, and $<$ Data $>$ element includes the non-XML format content, such as binary code. $<$ Manifest $>$ element specifies the list of the documents, and each $<$ Reference $>$ element corresponds to a document, namely the payload. This format is beneficial to the quick processing of the payload using the reference value without parsing the document. In ebMS, the main part of $<$ Body $>$ element is the $<$ Manifest $>$ element, which functions similarly to the cnMS.

\subsection{Interoperability Assurance Method}

As shown in the comparison result of cnMS and ebMS in section 4.1, the XML schema of the message format is quite similar between cnMS and ebMS. The similarity provides the basic for the transformation between cnMS and ebMS. It is very simple to do the bidirectional transformation using XSLT because cnMS and ebMS are both based on XML. An alternative approach is to add a receiving message processing module in the receiver system, to deal with the different message format, and achieve the interoperability in the messaging service layer.

For the interoperable e-Business solutions based on cnMS and ebMS, the interoperability can be ensured by standardized interoperability test. In the messaging service layer, the Interoperability test levels are divided into 6 levels as shown below [3]:

\section{Table 1. Interoperability test levels of messaging service}

\begin{tabular}{cl}
\hline Test Level & \multicolumn{1}{c}{ Description } \\
\hline T1 & One way messaging \\
T2 & Synchronized message reply \\
T3 & SSL security messaging \\
T4 & XML signature messaging \\
T5 & Reliable messaging \\
T6 & Error messaging \\
\hline
\end{tabular}

\section{Conclusion and Future Work}

The study of the Interoperability of Messaging Service layer provides the theoretical and technical basic for the interoperability of the fundamental layer of e-Business specifications, not only between cnXML and ebXML, but also between other XML-based eBusiness specifications. It also provides the proof for the messaging service interoperability test. With the IA of Messaging Service layer, Interoperability of business process, business term, namely the process and semantic interoperability layers, will be considered seriously. Our research will cover these fields in the future work. Semantic interoperability layer can be achieved by constructing a common ontology library with the support of domain experts, and the process interoperability layer can be achieved by the standardization of meta-process, such as BPSS activity. 


\section{References}

[1] Alan Kotok, David R.R.Webber, ebXML: The New Global Standard for Doing Business over the Internet, Macmillan Computer Publisher, New York, 2001.

[2] China e-Business Technology Research Center, "cnXML Tutorial", http://www.cnxml.org.cn/cnXML.htm, 2004 (in Chinese).

[3] Peng Liang, Keqing He, Bing Li, Jin Liu, "Interoperability Test of ebXML e-Business Solutions", Proceedings of the International Conference of Computer Information Technology (CIT2004), IEEE Computer Society, New York, 2004, pp. 1004-1007.

[4] Christof Bornhovd, "Semantic Metadata for the Integration of Web-Based Data for Electronic Commerce", Proceedings of the International Workshop on Advance Issues of E-Commerce and Web-Based Information Systems (WECWIS1999), IEEE Computer Society, New York, 1999, pp. 137-145.

[5] Ding Jun-hua, Dong Huan, Wu Ding-hao, "Researches on Software Interoperability", Journal of Computer Research \& Development, 1998, Vol 35(7) pp. 577-583 (in Chinese).

[6] Microsoft Corporation, "Web Services Interoperability and SOAP", http://msdn.microsoft.com/archive/enus/dnarxml/html/soapinteropbkgnd.asp, 2001.

[7] OASIS ebXML Technical Committee, "ebXML Technical Architecture Specification v1.04", http://www.ebxml.org/specs/ebTA.pdf, 2002.

[8] World Wide Web Consortium, "SOAP 1.2 Specification", http://www.w3.org/TR/soap12/, 2003.

[9] OASIS ebXML Technical Committee. "ebXML Messaging Service Specification v2.0", http://www.ebxml.org/specs/ebMS2.pdf, 2002.

[10] Zhang Wei, Ma Fan-yuan, "Study on Message Based on SOAP in cnXML", Journal of Computer Engineering and Application, 2003, Vol 12(17) pp. 14-17 (in Chinese). 